\title{
Monolithic MEMS position sensor for closed-loop high-speed atomic force microscopy
}

\author{
N. Hosseini ${ }^{\text {a }}$, A. P. Nievergelt ${ }^{\text {a }}$, J. D. Adams ${ }^{a}$, V. T. Stavrov ${ }^{b}$ and G. E. Fantner ${ }^{a}$ \\ ${ }^{a}$ Laboratory for Bio and Nano Instrumentation, School of Microengineering, Ecole Polytechnique \\ Federale de Lausanne, 1015 Lausanne, Switzerland \\ ${ }^{\mathrm{b}}$ AMG Technology Ltd., Microelectronica Ind. Zone, 2140 Botevgrad, Bulgaria
}

E-Mail: georg.fantner@epfl.ch

\begin{abstract}
The accuracy and repeatability of atomic force microscopy (AFM) imaging significantly depend on the accuracy of the piezoactuator. However, nonlinear properties of piezoactuators can distort the image, necessitating sensor-based closed-loop actuators to achieve high accuracy AFM imaging. The advent of high-speed AFM has made the requirements on the position sensors in such a system even more stringent, requiring higher bandwidths and lower sensor mass than traditional sensors can provide. In this paper, we demonstrate a way for high-speed, high-precision closed-loop AFM nanopositioning using a novel, miniaturized MEMS position sensor in conjunction with a simple PID controller. The sensor was developed to respond to the need for small, lightweight, high-bandwidth, long-range and subnm-resolution position measurements in high-speed AFM applications. We demonstrate the use of this sensor for closed-loop operation of conventional as well as high-speed AFM operation to provide distortion-free images. The presented implementation of this closed-loop approach allows for positioning precision down to $2.1 \AA$, reduces the integral nonlinearity to below $0.2 \%$, and allows for accurate closed loop imaging at line rates up to $300 \mathrm{~Hz}$.
\end{abstract}




\section{Introduction}

Piezoelectric actuators are extensively used in a variety of scanning applications such as scanning tunneling microscopes (STMs) and atomic force microscopes (AFMs) to move a sample or probe with nanometer resolution ${ }^{1,2}$. However, nonlinear behavior of piezoactuators, such as hysteresis, creep and thermal drift, significantly affects positioning precision and results in image distortion ${ }^{3,4}$.

To account for these nonlinearities, two general types of control approaches are used: openloop feed-forward input shaping or closed-loop feedback systems. In the first case, the appropriate piezo drive signal to linearize the motion of the scanner is calculated from a mathematical model ${ }^{5-8}$. This is a well-known and simple approach to control the drive signal, but linearization is often not perfect, since hysteresis is scan-size and scan-speed dependent.

Closed-loop feedback systems can address hysteresis, creep and drift and have therefore become the preferred method to reduce the nonlinearity of piezoactuators ${ }^{9-13}$, as thoroughly discussed in a review paper by Fleming ${ }^{14}$. By comparing the real position (measured by a low-noise, precise position sensor) with the demanded position, a closed-loop real-time controller compensates the errors between the two trajectories. However, accurate and timely measurement of the real position is required for adequate tracking performance. Depending on the performance of the position sensor and the implementation, closed-loop feedback can otherwise add additional noise, introduce additional nonlinearities or cause phase loss. Much work has been done to develop long-range, high-resolution, high-bandwidth, and highly linear position sensors. Nevertheless, combining all these properties in a single sensor is a challenging task ${ }^{14,15}$, and therefore a tradeoff has to be made based on the particular application. Position sensors that have been used for nanopositioning applications include capacitive sensors ${ }^{16}$, linear variable differential transformer (LVDT) ${ }^{17}$, piezoresistive and piezoelectric strain gauge ${ }^{18,19}$, optical ${ }^{20}$, interferometry ${ }^{21}$, electrothermal ${ }^{22}$ and giant magnetoresistance (GMR) ${ }^{23}$ sensors. Each of these sensor types has relative strengths and weaknesses for nanopositioning applications. Optical sensors provide high-resolution and high- bandwidth positioning capability but often require expensive components ${ }^{14}$. Non- 
optical sensors like capacitive sensors have high bandwidth and good resolution but have internal nonlinearity, which requires additional nonlinearity compensation ${ }^{14}$. Resistive strain gauges are simple and low cost, and can be bonded into the actuator, however the temperature sensitivity and nonlinearity need to be considered ${ }^{14}$. LVDT sensors provide high-resolution, large-range and linear positioning but suffer from a low bandwidth ${ }^{17}$. GMR sensors may provide high-bandwidth and high-resolution position sensing, but cannot be used in applications where magnetic fields can deteriorate imaging accuracy.

While these sensors perform well for normal-speed AFM, most are expensive, require nonnegligible forces, and/or add a significant amount of moving mass to the scanner. This added mass is an especially severe problem for high-speed AFMs, since the moving mass determines to a large extent the structural resonances and therefore the maximum achievable scan speed ${ }^{24}$. In this work, we present a small, lightweight and batch-fabricated microelectro-mechanical system (MEMS) position sensor ${ }^{25}$ implemented in closed-loop configuration in a standard as well as a high-speed AFM. This MEMS sensor uses mechanical deamplification flexures to adapt the sensor dynamic range to the scan-range of the scanner. These flexures are fabricated with sidewall-embedded piezoresistors for position sensing. The sidewall-embedded piezoresistors are inherently matched and result in intrinsic thermal stability as well as high sensitivity. These properties, combined with the low stiffness and low mass of the MEMS sensors, are essential to achieving the high resolution, low drift and high bandwidth of the closed-loop AFM scanner presented in this work.

\section{Experimental setup and controller implementation}

\subsection{Sidewall piezoresistive position sensor}

Closed-loop positioning performance depends considerably on the position sensor as the measuring device. To reduce the tracking error, the resolution, bandwidth and linearity of the sensor are primary concerns. Many SPM applications also require a relatively large range of motion (up to hundreds of micrometers), which is often achieved with a reduced resolution of 
the position sensor. In addition, the sensor should have high mechanical sensitivity and small thermal sensitivity for accurate measurement.

The presented piezoresistive MEMS strain sensor is based on recently developed sidewalldoped piezo-resistive strain sensors with mechanical deamplification for extended ranges ${ }^{25}$. Figure 1(a) illustrates the layout of one of the position sensors with $500 \mu \mathrm{m}$ range of motion;

(a)

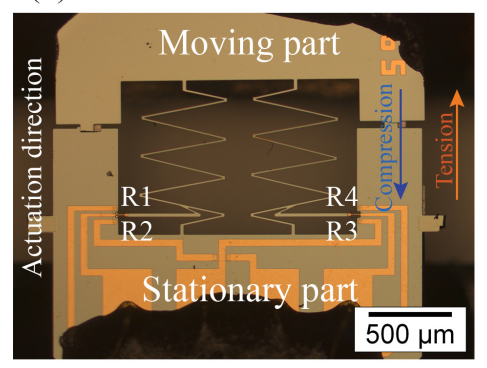

(d) Sensor in tension

_ Sensor in compression

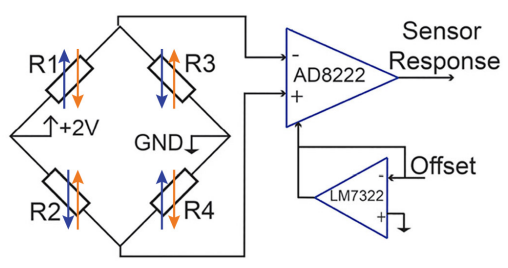

(b)

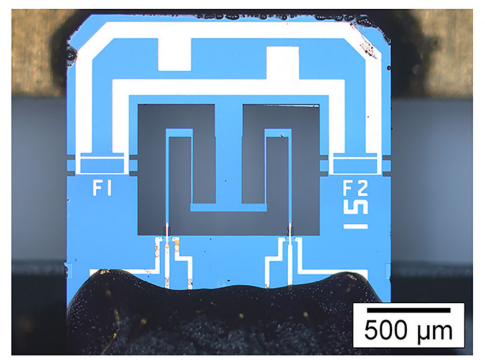

(c)

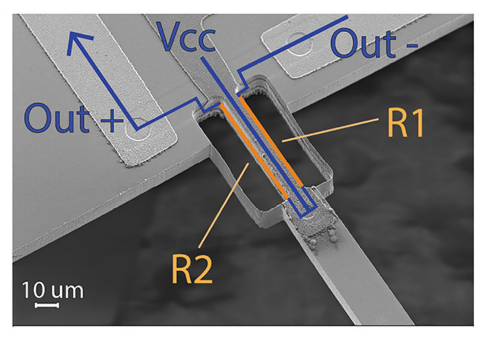

(e)

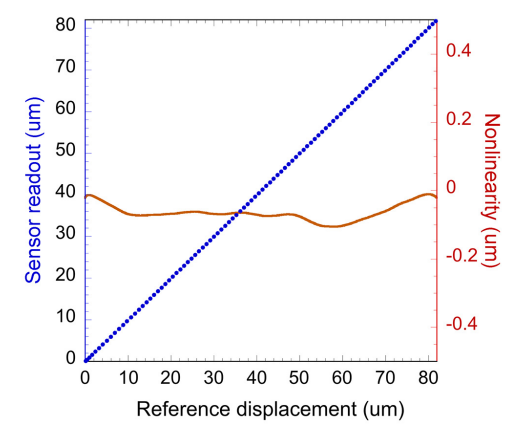

Fig. 1. (a) Optical image of the 500- $\mu \mathrm{m}$-range MEMS sensor. When $\mathrm{R}_{1}$ and $\mathrm{R}_{4}$ get compressed, $\mathrm{R}_{2}$ and $\mathrm{R}_{3}$ get elongated when the moving part moves upwards and vice versa. (b) Optical image of the sidewall piezoresistor $100 \mu \mathrm{m}$ MEMS sensor. (c) SEM image of one lever and two sidewall piezoresitors. (d) Instrumentation amplifier based readout and compensation offset for the resistor mismatch. The bridge bias voltage is 2 V. (e) Linearity measurement of the $500 \mu \mathrm{m}$ position sensor.

It comprises an outer frame and two compliant cantilevers with sidewall piezoresistors. Each cantilever is connected to the stationary and moving parts of the frame via a spring, which transduces the displacement of the moving frame to a strain in the area of Boron doped sidewall piezoresistors. Figure 1(b) shows an alternative sensor layout with $100 \mu \mathrm{m}$ motion range, where the linear displacement is transferred to a bending motion of the modified reed mechanisms. The sensor with $100 \mu \mathrm{m}$ scan range has higher sensitivity comparing to $500 \mu \mathrm{m}$ scan range sensor and provides higher nanopositioning resolution. (See supplementary material for videos of the action of the sensor.)

Four piezo-resistors are pre-wired on chip in a fully active Wheatstone-bridge configuration, where $R_{1}$ and $R_{4}$ change equivalently as do $R_{2}$ and $R_{3}$. Figure $1(c)$ shows the electrical 
pathway in one lever with two sidewall piezoresistors. The piezo-resistors are placed at the base of the flexure where they experience maximum strain in the beam. The cantilever beam is narrower there so that additional stress concentration occurs at the points of the piezoresistors.

The resistance change in the sidewall-embedded piezo-resistors is measured using Wheatstone bridge readout electronics based on a low-noise instrumentation amplifier (AD8222, Analog Devices, MA, USA); see figure 1(d). In the case of any temperature change, these four resistors experience the same amount of change and the Wheatstone bridge configuration compensates this undesired change.

One of the fundamental factors for closed-loop operation is the linearity of the position sensor, as any deviation is mapped onto the controlled position. For instance, capacitive sensors are inherently non-linear, which requires further measurement and calculation to linearize the response ${ }^{26}$. To test the linearity of the $500 \mu \mathrm{m}$ MEMS position sensor, we compared the sensor response with the motion of a precision, commercial 3D closed-loop positioning stage, having a closed loop range of $82 \mu \mathrm{m}$, (TRITOR 102, Piezosystem Jena). The resolution of the positioning stage is specified at $2 \mathrm{~nm}$ with a nonlinearity of $0.1 \%$. Figure 1(e) shows that the linearity of our $500 \mu \mathrm{m}$ position sensor tested over $82 \mu \mathrm{m}$ (the full range of the reference stage) is better than $0.15 \%$.

\subsection{Closed-loop implementation}

Piezotube scanners are very popular for AFM instruments, since they provide a large range of motion, have high resolution and can be easily used as 3D nanopositioners. The drawback of the tube design is its relatively low lateral stiffness, making it sensitive to the mass and loads that might be applied by position sensors. Many conventional nanopositioning sensors are therefore not suitable for use with piezo tubes. The sensor presented in this paper is compact and lightweight, with high displacement sensitivity and has a very low stiffness, and thereby does not restrict the motion of the piezo tube.

Here, we retrofitted a commercial $120 \mu \mathrm{m}$ sample scanner (J scanner, Digital Instruments, Santa Barbara, USA) with 500- $\mu \mathrm{m}$-range sidewall-embedded MEMS sensors for the $\mathrm{X}$ and $\mathrm{Y}$ 
axes respectively. In order to accurately position the sample, the position sensors are placed on top of the piezotube scanner as close as possible to the scanned sample. The moving part of the sensor is attached to a PCB that is glued on top of the scanner, and the stationary part is attached to a thin ring that is mounted around the piezo-guard (see figure 2(a)).

Figure 2 (b) describes the block diagram of the closed-loop implementation. A simple LabVIEW program is implemented on FPGA (NI CRIO 9074 FPGA). This program consists of two main modules. One is the PID controller and the other is a gain-shear correction. The measured sensor's signal is first compared with the low voltage drive signal from the AFM controller for each direction. These two signals need to have the same amplitudes, and therefore the gain correction is required prior to any subsequent step. Next, any nonorthogonally between $\mathrm{X}$ and Y displacements is corrected by modifying the sensor's signals as discussed in methods section 1.

The PID controller is a premade module in LABVIEW FPGA. The triangular reference trajectory from the AFM controller (Digital Instruments, Nanoscope 3a) is compared with the real position measured by the position sensors in the PI controller. The controller's gains are set manually by increasing the gains to the brink of oscillations. For imaging, the gains are then reduced slightly to avoid feedback oscillation in the system.

The PI controller compensates the error between the real position of the tube scanner and the triangular reference signal simultaneously. The compensated signal is amplified by a highvoltage amplifier and provides linear, drift- and creep-free displacement of the piezo tube scanner. To mechanically protect the sensors and facilitate the imaging, a sample holder with a small magnet was glued on top of the sensors and scanner.

The readout electronics are placed close to the sensors to minimize electrical noise pickup. Figure 2(c) displays the Multimode AFM, piezotube and the position sensor readout electronics. 

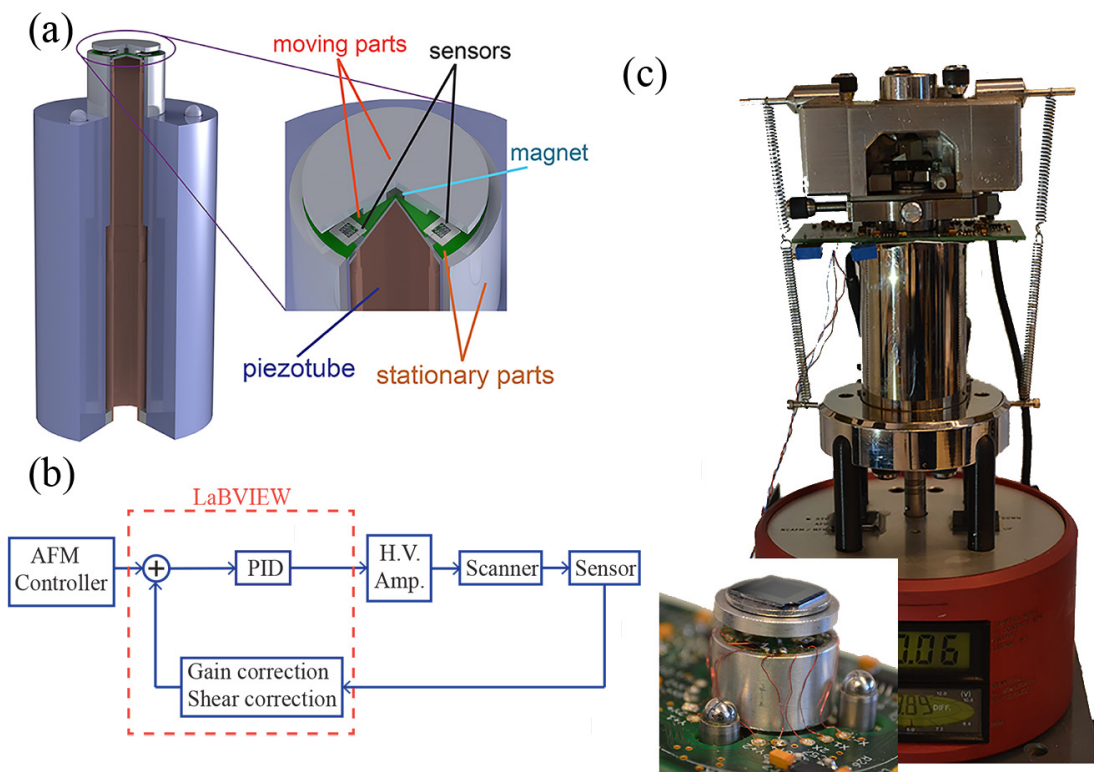

Fig. 2. (a) Schematic of the piezotube scanner and two position sensors. (b) Schematic of the closed-loop system. (c) Multi Mode AFM scanner retrofitted with position sensors and readout electronics.

\section{Implementation in a commercial AFM with a piezoelectric tube scanner}

In general, the non-ideal behavior of piezoactuators causes several kinds of artifacts in AFM imaging. Figure 3(a) demonstrates the position sensor response in open- and closed-loop systems while a triangular trajectory was applied to the piezoactuator. As is clear in the figure, there is a lag between the response and reference signal in open loop. However, in the closed-loop system, the PID controller compensates the position deviation due to inherent hysteresis of the piezoactuator.

To highlight this effect we overlaid two diagonally cropped images, one in open loop and one in closed loop. The nonlinearity caused by hysteresis in the slow scan axis is observable by comparing the image half taken while scanning downward with the one scanning upward in open loop (see figure 3(b)). On the other hand, closed-loop scanning compensates the undesired mismatch between images and provides repeatable scanning (see figure 3(c)). 
(a) Hysteresis compensation

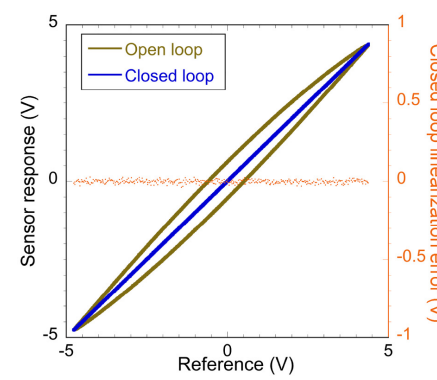

(b) Open loop nonlinearity

Downward scan direction (image 1)

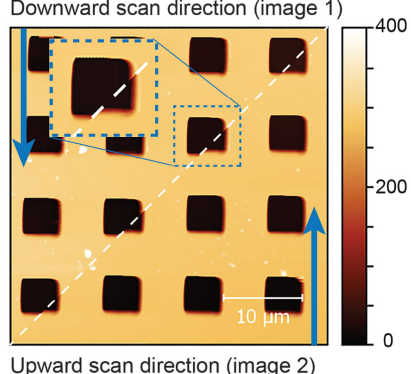

(c) Closed loop nonlinearity

Downward scan direction (image 1)

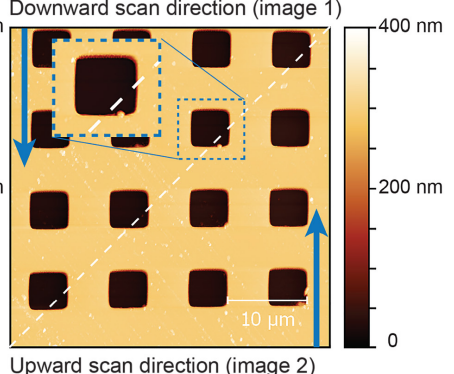

Fig. 3. Nonlinearity in open loop compared with closed-loop scanning. (a) Open-loop hysteresis and closed-loop linear

displacement of the piezotube scanner measured with the position sensor. (b) Two diagonally-stitched AFM images of a silicon

calibration grating in open-loop scanning where the mismatch between two images is observed. (c) Two diagonally stitched calibration-grating images in closed-loop scanning where the mismatch between two images is removed.

In comparison with capacitive sensors, there is no inherent nonlinearity in the presented sensor ${ }^{14}$. Another problem with open-loop piezo actuation is creeping, in which the piezo responds to a rapid positioning step by moving only a certain percentage quickly and then approaches slowly the rest of the way to the desired position. Creep in nanopositioners prevents accurate positioning when a sharp displacement is required, such as a scan offset. Figure 4(a) shows the creep in open-loop operation for a step offset. We imaged a $10 \mu \mathrm{m}$ pitch square silicon grating while the slow scanning axis was disabled (resulting in a repeated scanning of the same line, visible in the image as vertical stripes). We then added a $56 \mu \mathrm{m}$ offset to the fast scan axis. The open-loop scanner only performs part of the desired offset directly and then approaches the full offset very slowly, resulting in the bent stripes in the image figure 4(a). Closed-loop operation eliminates such creep, but only as far as the sensor does not exhibit any creep by itself. Strain-gauge-based nanopositioning sensors are known in particular to suffer from creep due to the required adhesion layer between the strain gauge and its substrate. While our position sensor also operates on a strain gauge, the sensing element and the flexure substrate are one monolithic silicon MEMS device, and therefore no adhesion layers are required. The stiffness of the flexure is also very low $(3 \mathrm{~N} / \mathrm{m})$, thereby adding no appreciable load to the piezo or its glue joints. Our closed-loop implementation therefore performs the $56 \mu \mathrm{m}$-offset step without exhibiting any detectable creep (see figure 4(b)). 
(a) 56 um offset in open loop

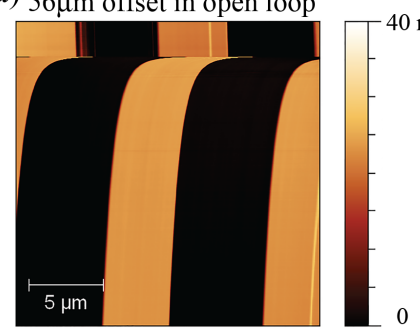

(b) $56 \mu \mathrm{m}$ offset in closed loop

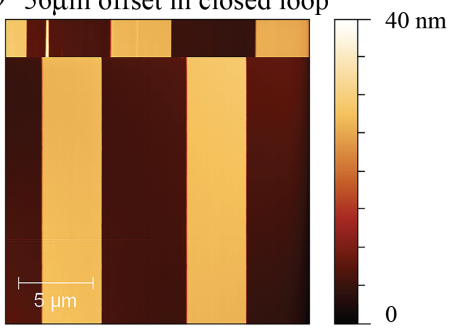

(c)

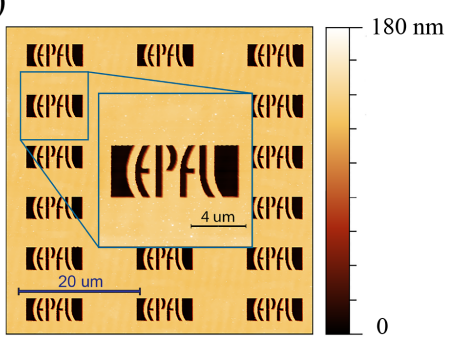

Fig. 4. Creep removal using a closed-loop system. (a) Nonlinear displacement of the piezoactuator according to $56 \mu \mathrm{m}$ applied offset in open loop due to creep. (b) Linear displacement of the piezoactuator according to $56 \mu \mathrm{m}$ applied offset in closed loop.

(c) Creep compensation permits accurate zooming in AFM imaging.

The lack of creep and the improved linearity of the closed-loop scanner allows for accurate zooming during AFM operation. Figure 4(c) demonstrates a $50 \mu \mathrm{m}$ scan size overview image with a dotted square depicting the chosen zoom area. The inset shows the zoomed in image showing exactly the chosen zoom area.

The achievable resolution of the positioning feedback system is influenced by the total noise of the position sensor over a given bandwidth ${ }^{27}$. The closed-loop resolution determines the distance between two distinguishable adjacent points and is defined in terms of the standard deviation of the positioning noise ${ }^{28}$. Nevertheless, there is a tradeoff between the positioning range, bandwidth and resolution of the position sensor ${ }^{7}$. To compare the lateral resolution in open- and closed-loop systems, we performed noise measurements in amplitude modulation AFM on a scanner equipped with the $100-\mu \mathrm{m}$-travel-range MEMS sensor. We used a sample of tilted mica, with the projected lateral noise calculated from the vertical amplitude signal in tapping mode (see Methods, section 2). The RMS values of the lateral noise in open loop and closed loop are $0.9 \AA$ and $2.1 \AA$ respectively. Figure 5 illustrates the normal distribution of the lateral noise in open and closed-loop systems. The measurement used a $1 \mathrm{kHz}$ bandwidth of the closed-loop system. 


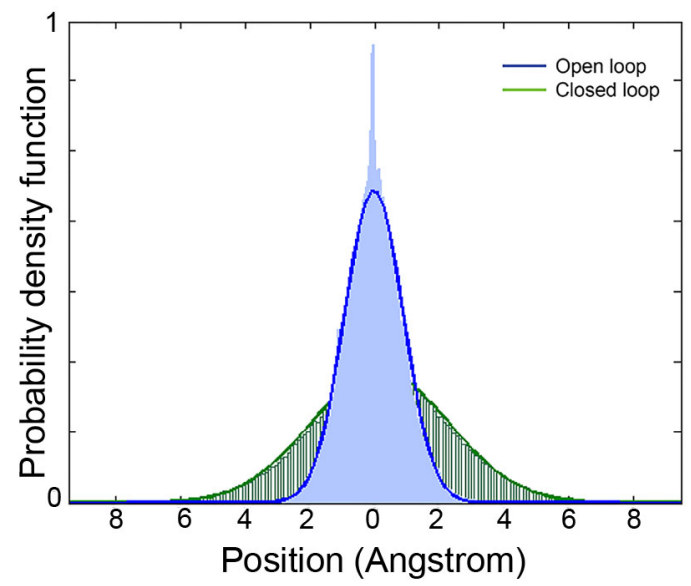

Fig. 5. Normal distribution of the lateral noise in open- and closed-loop systems. The $1 \sigma$ resolutions are $0.9 \AA$ and $2.1 \AA$ in open and closed loop respectively.

\section{Implementation in a custom high-speed AFM}

Much progress has been made in the area of high-speed AFM in recent years, with improvements for example in cantilevers ${ }^{29-31}$, detection systems ${ }^{30}$, controllers and scanner designs ${ }^{32,33}$. Obtaining high scanner resonance frequencies often requires very small and compact scanner designs ${ }^{34}$. Operating such high-speed scanners in closed-loop faces two additional challenges over conventional scanners: 1) a high sensor bandwidth with low noise density is necessary to maintain high-resolution closed-loop imaging over a larger frequency spectrum. 2) The size and mass of the sensor should not significantly increase the overall scanned mass, in order to not reduce the resonance frequency of the whole system ${ }^{35}$.

We upgraded our homebuilt piezo-stack high-speed scanner ${ }^{31}$ with two 100 - $\mu$ m-range position sensors to measure the lateral displacement (see figure 6(a)). The scanner is operated using a high-speed AFM controller (Anfatec AFT-MMC50, Germany) with a high-speed piezo amplifier (Techproject, Austria). We used the same FPGA as for the tube scanner implementation for closed-loop feedback. Figure 6(b) displays the X-axis frequency response of our home-built high-speed scanner measured with the MEMS sensor. The lateral resonance frequency of the scanner is $11.5 \mathrm{kHz}$, which is sufficiently high to image at several hundred lines/second. In order to minimize residual scanner vibrations at the turn around, a 
butterworth lowpass filter with a cut off frequency of $10 \mathrm{kHz}$ was implemented on the controller.

In order to measure the performance of the closed-loop high-speed system, we used the same down-up measurement as discussed earlier. To compare the implementation, images in open loop and closed loop are taken. Figure 6(c) displays two images taken in open loop where drift is visible between two images. Figure 6(d) includes two images in closed loop captured at $300 \mathrm{~Hz}$ scan rate and cut diagonally. The square pits have the correct regular spacing and the downward scanned image is well matched with the upward scanned image. At present, the sensors on our high-speed, closed-loop system are not fluid sealed, which limits the imagingin-fluid capabilities of this instrument for imaging biological samples. In order to provide a high-speed, closed loop scanner for biological samples, these sensors would need to be sealed or protected from potential fluid leaks.

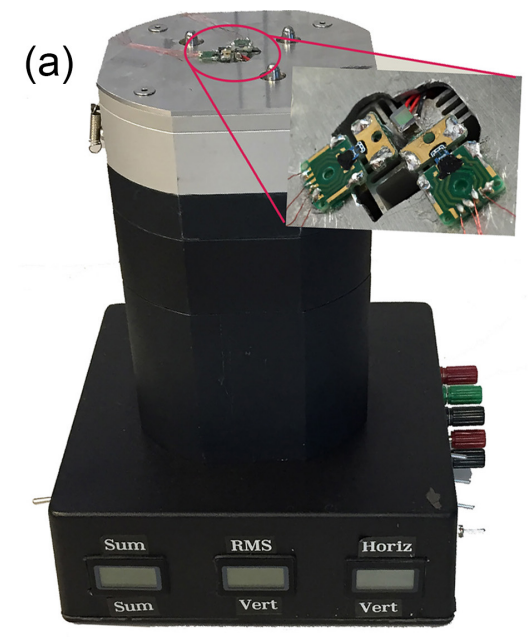

(c)

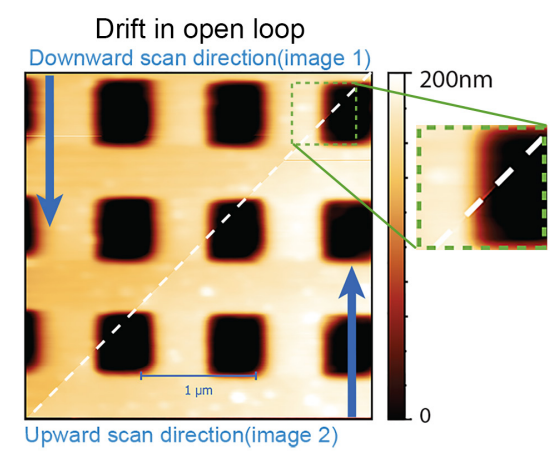

(b)
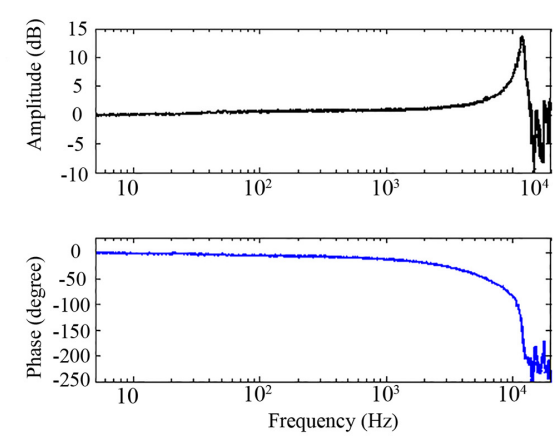

(d)

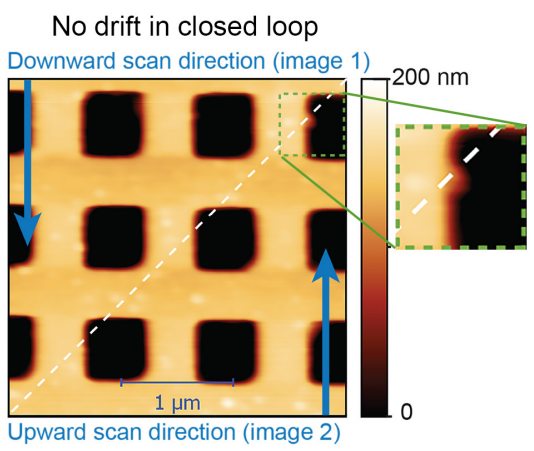

Fig. 6. High-speed closed-loop system. (a) Custom designed high-speed scanner and position sensors. (b) Frequency response of the high-speed scanner. (c) Drift in open loop (d) No nonlinearity at $300 \mathrm{~Hz}$ scan rate in closed-loop operation. 


\section{Discussion}

The performance of the sidewall piezoresistive position sensor shows great promise for lateral closed-loop AFM applications. The inherent linearity of the sensor simplifies implementation for electronics and signal processing. The position sensor's size and ease of use make it possible to install it in close proximity to the sample without impeding the scanner motion. The AFM images demonstrate adequate hysteresis, creep and drift removal in the implemented closed-loop system.

A drawback of our implementation is the need for crosstalk compensation between the $\mathrm{X}, \mathrm{Y}$, and to some extent Z-axis. Both the tube and flexure-based scanner implementations are parallel kinematic designs in the XY plane ${ }^{36}$. Therefore any motion in $\mathrm{X}$ will also lead to a motion in the $\mathrm{Y}$ axis, and vice versa. Additional cross coupling will occur in the sensors (coupling strength ca. 1:20 for $\mathrm{X}-\mathrm{Y}$ and 1:100 for $\mathrm{Z}-\mathrm{X}$ or $\mathrm{Y}$ ) in addition to inherent cross coupling of the scanners. This cross coupling depends on the exact design of the differential spring for the mechanical deamplification. We minimize this effect by aligning the sensor measurement axis very well with the scanner motion axis. Nevertheless, the crosstalk correction as well as the sensitivity correction can be easily implemented using standard shear transformations. For the tube scanner implementation, we implemented a cross-coupling correction for the Z-X and Z-Y signals using the low voltage actuation signal, which reduced any cross coupling below the measurement limit.

The presented sensor obtains its high sensitivity and linearity from the fact that it is a monolithic structure fabricated from single crystal silicon. However, this architecture, combined with the low spring constant of the MEMS flexures, also makes the sensor fragile. Therefore, great care must be taken not to break the lightweight, small and fragile sensor during mounting. In operation, we have not observed any fatigue failure or nonlinearity at the ends of the dynamic range. The sensor behaves linearly until it passes its allowed dynamic range, after which failure occurs in the form of brittle fracture in the silicon flexure. While we 
have not observed any failure of the sensors during months of operation, the sensors can become damaged when stretched beyond their dynamic range, for example when the closedloop controller becomes unstable and the scanner is excited at resonance.

The high bandwidth, small size, and low mass of the MEMS sensor make it well suited for high-speed AFM. The capacitive sensors often used in high-resolution closed-loop piezo stages are problematic for high-speed AFM applications, since they are comparatively large to the size of high-speed scanners and generally do not operate beyond a few kHz. Optical methods such as interferometry can easily operate at high frequencies and implementations are available in small form factors, but their high price limits their practical use. The advantage of the presented feedback system is the stability and high bandwidth due to small mass of the position sensors (combined with the scalability of batch fabrication). This performance enabled high-speed closed-loop imaging of our scanner with $300 \mathrm{~Hz}$ without instability in the system. Larger scan rates could be achieved by model-based filtering of the mechanical resonances of the home-built HS-scanner. We predict that further improvement of the readout electronics and direct implementation of the feedback in the AFM controller will reduce the closed-loop imaging noise to levels equal to the open-loop imaging noise.

\section{Conclusion}

In this work, we have presented a lateral feedback implementation using monolithic, sidewallembedded, MEMS-based nanopositioning sensors for both conventional piezo tube scanners as well as high-speed, flexure-based piezo scanners. Experimental results show distortion free, repeatable imaging with accurate zoom and offset operation. The small size, low noise and high bandwidth of the sensor combined with the simplicity of the readout electronics makes such a MEMS based closed-loop system ideally suited for integration into piezo-tube scanners as well as compact, high-speed, flexure-based AFM scanners. 


\section{Methods:}

\section{Closed loop implementation}

Shear and gain corrections are required before feeding the sensor's signal to PI controller. The gain correction is performed by comparing the sensor's signal with the low voltage drive signal from the AFM controller, and amplifying the sensor signal to have the same amplitude as the low voltage drive signal. The linearity of the sensor guarantees that once the gain is set there is no need to change it again. The gain correction is implemented by using high throughput multipliers in LABVIEW FPGA.

The shear correction is implemented as follows:

$\mathrm{X}_{\text {Sensor }}=\mathrm{X}_{\text {Sensor, Shear }}-\left(\alpha \times \mathrm{Y}_{\text {Sensor, Shear }}\right)$

$Y_{\text {Sensor }}=Y_{\text {Sensor, Shear }}-\left(\beta \times X_{\text {Sensor, Shear }}\right)$

Where $X_{\text {Sensor, Shear }}$ and $Y_{\text {Sensor, Shear }}$ are the sensor signals before shear correction, $X_{\text {Sensor }}$ and $Y_{\text {Sensor }}$ are sensor signals after shear correction. $\alpha$ and $\beta$ are the shear correction factors, which are defined to get orthogonal sensor's signals. The shear correction is performed by using high throughput multiplier and subtraction modulus in LABVIEW FPGA.

Afterwards the sensor signal is compared with the low voltage drive signal from the AFM controller. The PID module is chosen from "PID and fuzzy logic toolkit" in LABVIEW FPGA.

The PID gains are adjustable on the front panel of the LABVIEW code. The output of the PID is amplified with home built high voltage piezo amplifiers and then applied to the scanner.

\section{Lateral noise measurement}

The additional lateral noise in the closed-loop AFM image originates from the readout electronics, FPGA setup, the position sensor itself, as well as the existing position noise of the open-loop system. To characterize the total lateral noise in closed-loop imaging we recorded the amplitude variation on a tilted mica sample with a slope of $30^{\circ}$ in amplitude modulation 
mode, with a scan size of $0 \mathrm{~nm}$ while holding the position constant and using very low Zfeedback gains (just sufficient not to drift off the surface). The total noise consists of the vertical noise in $\mathrm{Z}$ and the lateral noise for each $\mathrm{X}$ and $\mathrm{Y}$ directions scaled by the $30^{\circ}$ angle. The lateral noise has been projected given the fact that the sample was tilted and has been compared for both open and closed-loop systems.

\section{Conventional AFM imaging}

We used a Bruker multimode in contact mode with retrofitted J scanner (digital instruments, Santa Barbara, USA). The low-voltage signals for X and Y scanning were outputs from the controller and reshaped through our closed-loop system. The compensated low voltage signals were then input to the controller.

\section{High-speed AFM imaging}

For high-speed AFM imaging we used an Anfatec controller (Anfatec AFT-MMC50, Germany) in high-speed contact mode. Our home built small lever head [27] was placed on our custom designed high-speed scanner [31] using a Bruker FastScan-C cantilever. Having the position sensors on the scanner, we implemented the closed-loop system on a LabVIEW FPGA as discussed in the main text.

\section{Acknowledgment}

The authors would like to thank Maja Dukic Pjanic and Pat McPhail from Bruker Nano Surfaces for helpful discussions, as well as the Atelier de l'institut de production et robotique at EPFL for fabrication of research equipment. This work has been funded by the European Union's Seventh Framework Programme FP7/2007-2011 under grant agreement 286146 and the European Union's Seventh Framework Programme FP7/2007-2013/ERC grant agreement 307338, Eurostars E!8213 - Triple-S, and the Swiss National Science Foundation through grants 205321134786 and 205320152675. 


\section{References}

1. Albrecht, T. R. \& Quate, C. F. Atomic resolution imaging of a nonconductor by atomic force microscopy. J. Appl. Phys. 62, 2599 (1987).

2. Binnig, G. \& Smith, D. P. E. Single-tube three-dimensional scanner for scanning tunneling microscopy. Rev. Sci. Instrum. 57, 1688 (1986).

3. Croft, D., Shed, G. \& Devasia, S. Creep, Hysteresis, and Vibration Compensation for Piezoactuators: Atomic Force Microscopy Application. J. Dyn. Syst. Meas. Control 123, 35 (2001).

4. Peng, J. \& Chen, X. A Survey of Modeling and Control of Piezoelectric Actuators. Mod. Mech. Eng. 03, $1-20$ (2013).

5. Clayton, G. M., Tien, S., Leang, K. K., Zou, Q. \& Devasia, S. A Review of Feedforward Control Approaches in Nanopositioning for High-Speed SPM. J. Dyn. Syst. Meas. Control 131, 061101 (2009).

6. Kenton, B. J. \& Leang, K. K. Design and Control of a Three-Axis Serial-Kinematic High-Bandwidth Nanopositioner. IEEE/ASME Trans. Mechatronics 17, 356-369 (2012).

7. Devasia, S., Eleftheriou, E. \& Moheimani, S. O. R. A Survey of Control Issues in Nanopositioning. IEEE Trans. Control Syst. Technol. 15, 802-823 (2007).

8. Su, C.-Y., Stepanenko, Y., Svoboda, J. \& Leung, T. P. Robust adaptive control of a class of nonlinear systems with unknown backlash-like hysteresis. IEEE Trans. Automat. Contr. 45, 2427-2432 (2000).

9. Tuma, T. et al. A high-bandwidth spintronic position sensor. Nanotechnology 25, 375501 (2014).

10. Fleming, A. J., Wills, A. G. \& Moheimani, S. Sensor Fusion for Improved Control of Piezoelectric Tube Scanners. IEEE Trans. Control Syst. Technol. 16, 1265-1276 (2008).

11. Rana, M. S., Pota, H. R. \& Petersen, I. R. Nonlinearity Effects Reduction of an AFM Piezoelectric Tube Scanner Using MIMO MPC. IEEE/ASME Trans. Mechatronics 20, 1458-1469 (2015).

12. Esbrook, A., Tan, X. \& Khalil, H. K. Control of Systems With Hysteresis Via Servocompensation and Its Application to Nanopositioning. IEEE Trans. Control Syst. Technol. 21, 725-738 (2013).

13. Liu, X., Tong, J. \& Sun, Y. Millimeter-sized nanomanipulator with sub-nanometer positioning resolution and large force output. Smart Mater. Struct. 16, 1742-1750 (2007).

14. Fleming, A. J. A review of nanometer resolution position sensors: Operation and performance. Sensors Actuators A Phys. 190, 106-126 (2013).

15. Tuma, T., Sebastian, A., Lygeros, J. \& Pantazi, A. The Four Pillars of Nanopositioning for Scanning Probe Microscopy: The Position Sensor, the Scanning Device, the Feedback Controller, and the Reference Trajectory. IEEE Control Syst. 33, 68-85 (2013).

16. Pinsopon, U., Cetinkunt, S. \& Nakajima, S. Design, fabrication, and real-time neural network control of a three-degrees-of-freedom nanopositioner. IEEE/ASME Trans. Mechatronics 5, 273-280 (2000).

17. Salapaka, S., Sebastian, A., Cleveland, J. P. \& Salapaka, M. V. High bandwidth nano-positioner: A robust control approach. Rev. Sci. Instrum. 73, 3232 (2002). 
18. Barlian, A. A., Park, W.-T., Mallon, J. R., Rastegar, A. J. \& Pruitt, B. L. Review: Semiconductor Piezoresistance for Microsystems. Proc. IEEE 97, 513-552 (2009).

19. Yong, Y. K., Fleming, A. J. \& Moheimani, S. O. A Novel Piezoelectric Strain Sensor for Simultaneous Damping and Tracking Control of a High-Speed Nanopositioner. IEEE/ASME Trans. Mechatronics 18, $1113-1121(2013)$.

20. Shan, Y., Speich, J. E. \& Leang, K. K. Low-Cost IR Reflective Sensors for Submicrolevel Position Measurement and Control. IEEE/ASME Trans. Mechatronics 13, 700-709 (2008).

21. Schneir, J. Design of an atomic force microscope with interferometric position control. J. Vac. Sci. Technol. B Microelectron. Nanom. Struct. 12, 3561 (1994).

22. Zhu, Y., Moheimani, S. O. R. \& Yuce, M. R. Simultaneous Capacitive and Electrothermal Position Sensing in a Micromachined Nanopositioner. IEEE Electron Device Lett. 32, 1146-1148 (2011).

23. Kartik, V. et al. High-bandwidth nanopositioner with magnetoresistance based position sensing. Mechatronics 22, 295-301 (2012).

24. Kindt, J. H., Fantner, G. E., Cutroni, J. A. \& Hansma, P. K. Rigid design of fast scanning probe microscopes using finite element analysis. Ultramicroscopy 100, 259-65 (2004).

25. Stavrov, V. T., Todorov, V. M., Shulev, A. A. \& Hardalov, C. M. MEMS sensors for mm-range displacement measurements with sub-nm resolution. in SPIE Microtechnologies (Schmid, U., Sánchez de Rojas Aldavero, J. L. \& Leester-Schaedel, M.) 87632G (International Society for Optics and Photonics, 2013). doi:10.1117/12.2017381

26. Kim, M., Moon, W., Yoon, E. \& Lee, K.-R. A new capacitive displacement sensor with high accuracy and long-range. Sensors Actuators A Phys. 130-131, 135-141 (2006).

27. Mohammadi, A., Yuce, M. R. \& Moheimani, S. O. R. Dealing with 1/f noise in MEMS electrothermal sensing. in IECON 2011 - 37th Annu. Conf. IEEE Ind. Electron. Soc. 4054-4058 (IEEE, 2011). doi:10.1109/IECON.2011.6119974

28. Fleming, A. J. \& Leang, K. K. Design, Modeling and Control of Nanopositioning Systems. (Springer International Publishing, 2014). doi:10.1007/978-3-319-06617-2

29. Adams, J. D. et al. High-speed imaging upgrade for a standard sample scanning atomic force microscope using small cantilevers. Rev. Sci. Instrum. 85, 093702 (2014).

30. Ando, T. High-speed atomic force microscopy coming of age. Nanotechnology 23, 062001 (2012).

31. Fantner, G. E. et al. Components for high speed atomic force microscopy. Ultramicroscopy 106, 881-7 (2006).

32. Aphale, S. S. \& Reza Moheimani, S. O. Design, Identification, and Control of a Flexure-Based XY Stage for Fast Nanoscale Positioning. IEEE Trans. Nanotechnol. 8, 46-54 (2009).

33. Moheimani, S. O. R. Invited review article: accurate and fast nanopositioning with piezoelectric tube scanners: emerging trends and future challenges. Rev. Sci. Instrum. 79, 071101 (2008).

34. Leang, K. K. \& Fleming, A. J. High-speed serial-kinematic SPM scanner: design and drive 
considerations. Asian J. Control 11, 144-153 (2009).

35. Wadikhaye, S. P., Yong, Y. K., Bhikkaji, B. \& Reza Moheimani, S. O. Control of a piezoelectrically actuated high-speed serial-kinematic AFM nanopositioner. Smart Mater. Struct. 23, 025030 (2014).

36. Dasgupta, B. \& Mruthyunjaya, T. S. Force redundancy in parallel manipulators: theoretical and practical issues. Mech. Mach. Theory 33, 727-742 (1998). 